\section{Diseño paramétrico. El gran desafío del siglo XXI}

Sandra Navarrete *

\begin{abstract}
Resumen: Estamos habituados a responder con el diseño a los nuevos modelos conceptuales que emergen de los cambios de paradigmas, de los teóricos y críticos, de las referencias epistemológicas internacionales. El diseño paramétrico se nos presenta en una situación diferente. De la complementariedad con otras disciplinas (de base eminentemente tecnológica), se ha generado una nueva forma de proyectar, atendiendo al proceso y sus múltiples variaciones, pero con escasa argumentación teórica. Se asiste a numerosos encuentros de esta temática en los que se cae en un éxtasis formal, que pareciera no necesitar mayores explicaciones. Pero, dado que el diseño está al servicio de la sociedad (no es arte autoreferencial), surge la imperante necesidad de encontrar el sustento conceptual a esta nueva modalidad de praxis.
\end{abstract}

Palabras clave: diseño inteligente - diseño paramétrico - diseño responsivo - materialidad - proyectual.

[Resúmenes en inglés y portugués en la página 72]

${ }^{(*)}$ Investigadora del Departamento de Investigaciones Científicas, Tecnológicas y Vinculación y Profesora Titular en la Universidad de Mendoza. Miembro del Comité Doctorado, Universidad de Palermo. Miembro del Comité Doctorado y Profesora Titular, en la Universidad Nacional de San Juan. Profesora Titular en la Universidad San Martín de Porres, Lima y en la Universidad Nacional de Cuyo.

Para que un objeto sea asequible al análisis no basta con darse cuenta de su existencia. Es necesario además que una teoría pueda aceptarlo.

En la relación entre teoría y experiencia, es siempre la primera quien inicia el diálogo.

Es la teoría la que determina la forma de la pregunta, es decir, los límites de la respuesta (Samaja, 1999).

Así como el siglo XX comenzó con el auge de la industrialización, del mismo modo, el inicio del siglo XXI propone nuevos desafíos, tales como la era digital, la responsabilidad social, ecológica, medioambiental. Ante este nuevo escenario, los procesos de diseño tienen 
la urgente necesidad de revisar sus bases conceptuales y procedimentales, incorporando el aporte de otras disciplinas.

El diseño paramétrico es un recurso innovador, aplicable a diferentes escalas: al diseño industrial, a la arquitectura y al urbanismo.

En el enfoque paramétrico el diseñador comienza por establecer las relaciones entre las partes, construye su diseño a partir de estas relaciones y modifica estas relaciones a partir de la evaluación y selección de los resultados obtenidos.

De esta manera se potencia la posibilidad de examinar variantes sin la necesidad de rehacer cada vez el trabajo de representación. Esto exige un cambio en los hábitos de trabajo del diseñador que debe incluir la definición de las relaciones, de la lógica que hace coherente su diseño, como fase imprescindible en su proceso de diseño. Dentro de un modelo paramétrico, cada entidad posee parámetros asociados. Estos parámetros controlan las diversas propiedades geométricas de la entidad, tales como su longitud, anchura, altura, radio, etc. También controlan la ubicación de estas entidades en el modelo y cómo las entidades se relacionan entre sí. Los parámetros pueden ser modificados por el operador para crear la geometría deseada.

En el proceso proyectual tradicional se utiliza el método top down, que significa el tener una "receta" para lo que se va a hacer, es decir, una forma preestablecida, que se somete a una realidad o a un orden.

El diseño paramétrico ocupa el método botton up, que significa crear una lógica a partir de relaciones específicas, que se pueden ir modificando según los requerimientos sociales y contextuales.

La forma habitual de trabajar con software como el AutoCAD, hasta ahora ha sido la de crear entidades geométricas a partir de las cuales se representa un diseño preconcebido. El procedimiento que se utiliza requiere una gran destreza en el uso de la herramienta informática, especialmente para llegar a la tridimensionalidad, y presenta grandes limitaciones en diseños que escapan de las formas geométricas tradicionales. Esta restricción llevó a nuevas propuestas digitales, más versátiles de fácil acceso al "proceso" de diseño.

\section{¿Qué es un parámetro?}

En diferentes publicaciones de repercusión mundial se está hablando de diseño paramétrico, los estudiantes, ante desconcertados ojos de docentes, están ganando concursos internacionales, pero la participación en encuentros sobre este nuevo procedimiento proyectual deja más dudas que certidumbres. Se hace necesario entonces, indagar en la esencia de esta propuesta hipermoderna, intentando comprender lo que hasta ahora se maneja de modo intuitivo...

"Pensamientos sin contenidos, son vacíos; Intuiciones sin conceptos, son ciegas" (Immanuel Kant).

El primer acercamiento a esta problemática, impone una definición de conceptos, reconocer los contenidos esenciales, más allá del juego formal, intuitivo, que los sistemas informáticos actuales nos permiten. 
Un parámetro es un dato que es tomado como necesario para analizar o valorar una situación. A partir del parámetro, una determinada circunstancia puede entenderse o situarse en perspectiva.

Para las matemáticas, un parámetro es una variable que permite identificar, en una familia de elementos, a cada uno de ellos mediante su valor numérico.

Un parámetro estadístico es una función definida sobre los valores numéricos de una población. Se trata, por lo tanto de un valor representativo que permite modelizar la realidad.

\section{Un antecedente: el lenguaje de patrones}

"El empírico es el amante de los hechos en su íntegra multiplicidad, sin pulimento.

El racionalista es el adorador de principios abstractos y eternos." (Carl Gustav Jung)

No es nuevo que los diseñadores empíricos, que van transformando los procesos de diseño a partir del juego prueba-error, hayan intentado en distintos momentos de la historia, dar un paso sustentado por la mera experiencia. Tal como lo expresa la cita de Jung, son "amantes de la multiplicidad" y esto es lo que promete el diseño paramétrico, ilimitadas opciones, modificando variables. Pero también la cita dice "sin pulimento", es decir que estamos en el estadío de exploración, se hace necesario comenzar a abordar esta innovación con mayor precisión. Además Jung enuncia que "el racionalista es adorador de principios abstractos y eternos", sin ánimo de llegar a una postura tan extrema, en esta reflexión se pretende encontrar "principios" que sean comprensibles y transmisibles racionalmente.

En otros términos, el procedimiento de diseño por parámetros, lo había propuesto Alexander en el período de posguerra, usando la palabra "patrones".

Los patrones se utilizan para dar forma a valores de decisiones cuya efectividad resulta obvia a través de la experiencia (programa de necesidades, mostrados en los gráficos), pero que son difíciles de documentar.

Christopher Alexander acuñó el término lenguaje de patrón. Lo usó para referirse a los problemas normales del diseño arquitectónico y civil. Su uso iba desde la forma de estructurar una ciudad a cómo debían disponerse las ventanas en una habitación. La idea se popularizó gracias a su libro A Pattern Language. Alexander da la siguiente definición de patrón:

Cada patrón describe un problema que ocurre una y otra vez en nuestro entorno, para describir después el núcleo de la solución a ese problema, de tal manera que esa solución pueda ser usada más de un millón de veces sin hacerlo ni siquiera dos veces de la misma forma (Alexander, 1964).

Durante varias décadas este sistema fue aplicado en la enseñanza de la arquitectura, intentando reducir la complejidad de una obra, a sus aspectos funcionales (programa de necesidades). Era un modo de controlar los resultados, a través de grillas con un sistema de relaciones entre las distintas unidades de uso, evitando superposición de áreas, de circulaciones... evitando la incertidumbre. 
Un procedimiento similar fue el de la zonificación, que implicaba unir los locales de funciones similares (diurnas, nocturnas, servicios, etc.) en "zonas" independientes, interrelacionadas.

Para James Coplien un buen patrón debe cumplir los siguientes requisitos:

- Debe solucionar un problema: los patrones capturan soluciones, no sólo principios abstractos o estrategias.

- Son conceptos probados: los patrones proponen soluciones que han sido probadas, no teorías o especulaciones.

- La solución no es obvia: la mayoría de las técnicas de resolución de problemas (tales como métodos de diseño) intentan derivar soluciones partiendo de principios básicos. Los mejores patrones generan una solución para un problema indirectamente, una aproximación necesaria para los problemas de diseño más complejos.

- Describe una relación: los patrones no deben describir módulos, sino que deben describir sistemas, estructuras o mecanismos más profundos.

- El patrón debe tener un componente humano importante: todo software sirve para el confort humano o para la calidad de vida; los mejores patrones recurren explícitamente a la estética y a la utilidad.

Muchos patrones forman un lenguaje. Así como las palabras deben tener una relación gramática y semántica entre ellas para crear un lenguaje oral útil, los patrones de diseño deben estar relacionados unos con otros para poder formar un lenguaje de patrones. En el trabajo de Alexander está implícita la idea de que los patrones deben estar organizados en estructuras lógicas o estructuras intuitivas. La estructura (jerárquica, iteractiva, etc.) puede variar, dependiendo del tema. Cada patrón debe indicar su relación con otros patrones y con el lenguaje en sí.

Entonces, si se quiere diseñar paramétricamente un "espacio de circulación", se tendría que hacer una definición de variables (parámetros) y de sus indicadores medibles, como tipos de usuarios, cantidad de usuarios, flujos, tamaño de flujos, horas, por donde sigue el flujo, etc., y según esos parámetros y de sus distintos grados, se arma una forma.

\section{Indagación conceptual de la complejidad}

Frente a la complejidad de la sociedad actual, aparecen teorías que contemplan una dinámica no lineal, la teoría del no-equilibrio y la teoría del caos. Edgar Morin la toma como punto de partida para la elaboración de su perspectiva sobre la epistemología de la complejidad, la cual servirá de amplia aplicación en el campo de las ciencias sociales y de la educación. Para Morin la sociedad es un sistema global uno y complejo a la vez, dispone de calidades originales, no puede reducirse a la suma de los individuos que la constituyen. Es en este ámbito donde se inserta la expresión pensamiento complejo, concebida como el pensamiento que trata con la incertidumbre y es capaz de concebir la organización. La turbulencia del pensamiento no es un movimiento repetitivo sino en espiral, hay regeneración después de cada nueva adquisición, modificación o turbulencia encontrada, y de 
allí emerge el producto del pensamiento: lo conocido se transforma en concebido, es decir, en pensamiento.

Durante siglos, se buscaron reglas de composición que garantizaran un resultado estéticamente perfecto (ideal griego). Trabajar con la incertidumbre genera un alto grado de ansiedad en los diseñadores, ya que el resultado final es difícil de prever. En su análisis de la Edad Moderna, Morin sitúa a Pascal como el pensador clave de la complejidad, Hegel quien con su dialéctica anuncia la dialógica, especialmente importante es Nietzsche que provoca la crisis de los fundamentos de la certeza y finalmente Adorno, Horkheimer y Lukacs que aportan numerosos elementos de una crítica de la razón clásica y elementos para una concepción de la complejidad.

Morin señala una situación paradójica: se han adquirido muchos conocimientos sobre el mundo físico, biológico, psicológico, sociológico; han predominado los métodos de verificación empírica en la ciencia; en nombre de la razón se creyó enterrar mitos y tinieblas, y sin embargo el error, la ignorancia, la ceguera progresan por todas partes al mismo tiempo que los conocimientos. La inteligencia ciega, los errores, la ignorancia, no reconocen ni aprehenden la complejidad de lo real pues organizan el conocimiento de modo parcial, por ello se necesita de un pensamiento complejo.

Complejidad es lo que está tejido en conjunto: es un tejido de constituyentes heterogéneos inseparablemente asociados, que presenta la paradoja de lo uno y lo múltiple. Es también el tejido de eventos, acciones, interacciones, retroacciones, determinaciones, azares que constituyen nuestro mundo fenoménico (Arancibia y Philosophica: Enciclopedia filosófica on line, 2010).

En el área de las ciencias duras, especialmente en la rama de las matemáticas, la física, se habla de la Teoría del caos, que es la denominación popular de ciertos tipos de sistemas muy sensibles a las variaciones en las condiciones iniciales. Pequeñas variaciones en dichas condiciones iniciales pueden implicar grandes diferencias en el comportamiento futuro; complicando la predicción a largo plazo. Esto sucede aunque estos sistemas son en rigor determinísticos, es decir; su comportamiento puede ser completamente determinado conociendo sus condiciones iniciales.

\section{El aporte imprescindible de la informática}

En las ciencias de la computación, un parámetro es una variable que puede ser recibida por una rutina o una subrutina (que utilizan los valores asignados a un parámetro para modificar su comportamiento en el tiempo de ejecución).

La arquitectura paramétrica se puede definir de manera sencilla como una nueva forma de entender el proyecto y el diseño de arquitectura, que se beneficia con las nuevas tec- 
nologías informáticas de diseño automático. En cuanto a software específico, aparecen programas como rhinoceros y grasshopper.

Lo paramétrico está relacionado con conceptos como el crecimiento y las estructuras evolutivas (de hecho se puede hablar de diseño evolutivo).

La utilización de parámetros y la automatización de algunas tareas abren infinitas posibilidades de diseño; en una visión de la arquitectura en que las tecnologías puedan ejecutar, de manera eficaz, tareas que antes eran propias del arquitecto-diseñador. El arquitecto Ion Cuervas Mons, muestra una desafiante idea de arquitectura paramétrica aplicada a la participación de los ciudadanos. Menciona que se podría imaginar un software que permita a todo el mundo diseñar su propia vivienda, simplemente cambiando algunos parámetros según los deseos de cada uno.

\section{Acerca del método}

Si de método se trata, es necesario remontarse al origen de este concepto racional. El "Discurso del método" (Descartes, 1974), es una obra que pretende dar a conocer el método para poder llegar al conocimiento verdadero y encontrar la verdad. Fue escrito por René Descartes en 1637, este tratado es una de sus obras más importantes, considerada como una de las primeras obras de la filosofía moderna moderna. A pesar del tiempo transcurrido, sus afirmaciones hoy vuelven a ser vigentes. Descartes habla acerca del método que ha logrado desarrollar para elevar y aumentar sus conocimientos de forma gradual y paulatina, menciona por propia experiencia los beneficios que le ha proporcionado este método. Aunque él mismo hace una advertencia al lector: “... mi propósito no es enseñar aquí el método que cada uno ha de seguir para conducir bien su propia razón, sino tan solo permitir ver de qué manera he tratado de conducir la mía" (Descartes, 1974). Descartes en la última parte de su tratado nos habla acerca de la utilidad de la ciencia. La sexta y última supone un análisis de la investigación científica en general y en ella el autor confía en la necesidad de una comunidad científica que permita extender los conocimientos, así como en la necesidad de cultivar la salud del propio cuerpo para poder pensar adecuadamente.

\section{¿Cómo realizar un diseño paramétrico?}

Los integrantes de Chido Studio, una plataforma de investigación y exploración de diseño paramétrico y generativo, han sistematizado la información para poder explicar estos nuevos lenguajes de programación, aplicables a las estrategias de diseño y producción digital. Chido Studio, que está realizando workshop en diferentes lugares del mundo, propone el siguiente proceso. 
A partir de una idea abstracta (proveniente del mundo racional, no empírico) se proponen condiciones geométricas y matemáticas, factibles de ser traducidas al lenguaje informático. Se determinan las condiciones necesarias y se realizan pruebas con variables denominadas parámetros. Una vez seleccionados los parámetros (internos y externos - contextuales) se realiza una programación del proceso, que implica manipular los parámetros dimensionales, para lograr el producto que más se relacione con las premisas de diseño establecidas. Es un juego informático interminable, ya que cada variación va arrojando resultados muy diferentes. Y como clave de este tipo de diseño, es indispensable encontrar la forma de representación gráfica que más explicite el proceso.

Los objetivos que se proponen a priori en el proceso de diseño paramétrico son:

-Diseñar un proceso y no un resultado concreto: Al diseñar un proceso se desarrollan relaciones matemáticas y geométricas creando procesos y sistemas (algoritmos), los cuales permiten explorar más de un resultado, con ciertas premisas de diseño establecidas previamente.

- Posibilidad de relacionar variables / parámetros: Teniendo un proceso de diseño y no una forma preestablecida se pueden manipular sus variables y propiedades, las cuales se pueden modificar en tiempo real y así comparar resultados, con la finalidad de tener un producto final más eficiente.

- Resultados paramétricos y /o responsivos a condiciones establecidas previamente: A partir del diseño paramétrico se puede generar diseños inteligentes y/o responsivos estableciendo un criterio de diseño (exploración de formas), permitiendo adaptarse a cualquier situación, contexto, tectónico, etc. Es decir se puede adaptar el diseño a cualquier parámetro / variable que sea integrado al proceso de diseño, dando un resultado inteligente y responsivo que logra satisfacer un problema específico.

\section{La relación entre lo responsivo y la naturaleza}

Esta propuesta no estaría a la vanguardia, si no contemplara los aspectos indispensables de la realidad actual, ya asumidos como parte del paradigma de la neomodernidad.

El diseño no puede obviar las necesidades de preservación ecológica, la sustentabilidad, y por lo tanto la observación de la inteligente conducta de los seres vivos.

La piel humana y vegetal actúan, dando "respuestas" a contextos específicos (responsivo es capaz de dar respuestas). En particular el poro, que sirve para regular la humedad interior e intercambiar aire y vapor con el entorno, para controlar el cuerpo. El sudor, el grado de abertura del poro, la respiración.

Algunos diseño arquitectónicos, de cubiertas y cerramientos (la pile del edificio) se realiza teniendo en cuenta los efectos climáticos propios del lugar, así, las aberturas se orientan hacia las mejores posibilidades de asoleamiento, variando su estética por razones "responsivas". 
El ojo, y por extensión, el diafragma de una cámara fotográfica, sirven para regular la intensidad y calidad de luz recibida. Se trata de una manipulación a través, de nuevo, del grado de abertura y obturación, para manipular una imagen, la luz.

Si se considera que la naturaleza tiene una inteligencia propia, este paralelismo es un paso directo a la "inteligencia artificial", es decir al control automatizado informáticamente de la arquitectura, lo que actualmente se llama domótica.

\section{Búsqueda de opciones para la materialidad}

El diseño paramétrico no es una propuesta que apunte solamente a lo formal (como los primeros bocetos digitales de Zaha Hadid). Es integral, por lo que la materialidad es uno de los parámetros que deben tenerse en cuenta en el proceso.

De la era industrial a la digital, con la multiplicidad de recursos tecnológicos disponibles, este modelo proyectual se fija ciertas premisas:

- Fabricación digital: Una de las ventajas del diseño paramétrico es que permite integrar la fabricación digital directamente al diseño, ya que se integra la producción digital por medio de máquinas de control numérico o impresoras 3D.

Así es como con la producción digital se optimiza el tiempo y costos de producción, ya que aplica los conceptos básicos de la prefabricación.

Acerca de COMPO-SECTION, trabajo experimental de alumnos de la Universidad de Sevilla, podemos distinguir:

\section{Componente básico de la estructura}

La estructura se conforma mediante distintas piezas cuyas formas dependen del diseño que se haya elegido.

\section{Uniones}

El prototipo diseñado se caracteriza por un ensamblaje ortogonal de piezas de distintas morfologías que se unen entre sí a través de una serie de hendiduras, de manera que las dos placas que se intersectan tengan la misma altura en el punto de unión.

\section{Materiales}

El prototipo está hecho con cartón de proyectos de $3 \mathrm{~mm}$ de espesor. Sin embargo, para hacer un proyecto real, sería conveniente utilizar un material plástico resistente, o incluso metacrilato, lo que daría un interesante juego de luces debido a la transparencia del material.

\section{Diseño}

Se creó un modelo en CAD mediante curvas con la orden Spline, de tal manera que dos curvas perpendiculares entre sí se unan en un punto determinado. Una vez se tienen todas las curvas, es posible modificar la altura de los puntos de unión sin tener que cambiar el 
resto de la estructura. Dependiendo de la curvatura que se le quiera dar al conjunto, se decidió la separación entre lamas, de manera que en las zonas en las que la curva es más pronunciada la separación es menor.

\section{Sistema/lógica de crecimiento}

La estructura está pensada para un diseño acabado, por lo que no es posible que siga creciendo una vez se haya hecho el objeto inicial. Por otro lado, el tamaño del diseño no está limitado, por lo que cualquier proyecto es posible independientemente de su dimensión; podría utilizarse para la creación de muebles, paramentos, mobiliario urbano, elementos ornamentales, etc.

\section{Usos posibles en mobiliarios Estrategia de montaje}

Las piezas van encajadas entre sí mediante hendiduras, de tal manera que no es necesario el uso de ningún tipo de pegamento. Para mayor precisión de corte es conveniente utilizar la Cortadora Láser.

\section{Conclusión}

Una de las mayores ventajas del diseño paramétrico es la simbiosis entre disciplinas, la cual nos permite integrar criterios estructurales, sociales, simulaciones de flujo, etc. Esta necesidad de integrar disciplinas diversas es producto de la complejidad de la sociedad actual, a la que únicamente se le puede dar respuesta integral, integrando enfoques.

Hasta ahora el diseño paramétrico se presenta como una opción de experimentación proyectual, por las dificultades y costos que representa su materialidad, con la finalidad de que el modelo tridimensional no sea solo una maqueta virtual sino una herramienta capaz de darnos resultados e información para lograr diseños más reales que proponen resultados contundentes.

El desafío actual, de esta era que ha logrado un hábil manejo de los sistemas informáticos y de la tecnología industrial, es poner al servicio de la sociedad, esta propuesta de diseño inteligente.

\section{Bibliografía}

Alexander, C. (1964). Notes on the synthesis of forms. Harvard University Press.

Arancibia, M. D., (2010). Complejidad y Organizaciones. San Juan: Editorial Fundación Universidad Nacional de San Juan.

Bauman, Z. (2008). Mundo consumo. Etica del individuo en la aldea global. Paidos. España. Bertalanffy, L. von, (2003). Teoría General de los Sistemas. Fundamentos, desarrollo y aplicaciones, Fondo de Cultura Económica, México. 
Coplien, J. O. (1998). A Pattern Definition - Software Pattern. Addison Wesley. Descartes, R. (1974). Discurso del Método. España: Ed. Bruguera.

Lewin, R. (2002). Complejidad. El caos como generador del orden. Barcelona: Tusquets.

Morin, E. (2004). Introducción al pensamiento complejo. Madrid: Gedisa.

Ott, E. (2002). Chaos in Dynamical Systems. New York: Cambridge University Press.

Samaja, J. (1999). Epistemología y metodología. Buenos Aires: Editorial EUDEBA.

Tedeschi, A. (2010). Architettura parametrica: introduzione a Grasshopper. Potenza: Le

Penseur.

Summary: We are used to respond to the new design concept models emerging from the paradigm shifts of theorists and critics of international epistemological references. Parametric design is presented in a different situation. Complementarity with other disciplines (eminently technological based), has created a new way of designing, attending to the process and its many variations, but with little theoretical argument. It attends many meetings where this issue falls into a formal ecstasy that seems not to need further explanation. But, since the design is at the service of society (not self-referential art), arises the urgent need to find conceptual support to this new form of praxis.

Keywords: intelligent design - materiality - parametric design - project planning - responsive design.

Resumo: Estamos habituados a responder com o design aos novos modelos conceptuais que emergem das mudanças de paradigmas, dos teóricos e críticos, das referencias epistemológicas internacionais. O design paramétrico se apresenta numa situação diferente. Da complementaridade com outras disciplinas (de base eminentemente tecnológica), se gerou uma nova maneira de projetar, atendendo ao processo e suas múltiplas variações, mas com pobre argumentação teórica. Assiste-se a numerosos encontros desta temática nos quais se cai num êxtase formal, que pareceria não precisar explicações. Mas, como o design está ao serviço da sociedade (não é arte auto-referencial) surge a necessidade de encontrar o sustento conceitual para esta nova modalidade de práxis.

Palavras chave: design inteligente - design paramétrico - design responsivo - materialidade - projetual. 